\title{
Super Unit Cells in Aperture-Based Metamaterials
}

\author{
Dragan Tanasković, Zoran Jakšić, Marko Obradov, and Olga Jakšić \\ Centre of Microelectronic Technologies, Institute of Chemistry, Technology and Metallurgy, University of Belgrade, \\ Njegoševa 12, 11000 Belgrade, Serbia \\ Correspondence should be addressed to Zoran Jakšić; jaksa@nanosys.ihtm.bg.ac.rs
}

Received 4 December 2014; Accepted 12 January 2015

Academic Editor: Shuangxi Xing

Copyright ( $\odot 2015$ Dragan Tanasković et al. This is an open access article distributed under the Creative Commons Attribution License, which permits unrestricted use, distribution, and reproduction in any medium, provided the original work is properly cited.

\begin{abstract}
An important class of electromagnetic metamaterials are aperture-based metasurfaces. Examples include extraordinary optical transmission arrays and double fishnets with negative refractive index. We analyze a generalization of such metamaterials where a simple aperture is now replaced by a compound object formed by superposition of two or more primitive objects (e.g., rectangles, circles, and ellipses). Thus obtained "super unit cell" shows far richer behavior than the subobjects that comprise it. We show that nonlocalities introduced by overlapping simple subobjects can be used to produce large deviations of spectral dispersion even for small additive modifications of the basic geometry. Technologically, some super cells may be fabricated by simple spatial shifting of the existing photolithographic masks. In our investigation we applied analytical calculations and ab initio finite element modeling to prove the possibility to tailor the dispersion including resonances for plasmonic nanocomposites by adjusting the local geometry and exploiting localized interactions at a subwavelength level. Any desired form could be defined using simple primitive objects, making the situation a geometrical analog of the case of series expansion of a function. Thus an additional degree of tunability of metamaterials is obtained. The obtained designer structures can be applied in different fields like waveguiding and sensing.
\end{abstract}

\section{Introduction}

Nanocomposites based on two-dimensional arrays of subwavelength apertures in conductive material represent probably the most important class of metamaterials for the optical wavelength range. An example of such artificial structures is the fishnet metamaterials, which are the structures of choice for achieving negative value of effective refractive index in the optical range [1-3]. Another example is the extraordinary transmission arrays [4-6], where subwavelength apertures ensure, contraintuitively, an almost $100 \%$ optical transmission in a wavelength range determined by the surface plasmon resonance effects.

The function of these aperture-based metamaterials is based on the electromagnetic interplay between metal and dielectric parts in their composite periodic structures, where the dimensions of the features and the sizes of their unit cells are subwavelength. The fact that the radiation in the operating wavelength range is much larger than the structural details ensures the possibility to describe them by the effective values of their optical parameters [7]. In the ranges where the effective value of relative magnetic permeability and the relative dielectric permittivity are simultaneously below zero, the effective refractive index, being equal to the square root of the permittivity-permeability product, will be below zero too $[8]$.

One can observe subwavelength metal-dielectric composites from a more general point of view, where it can be said that the possibility to tailor their geometry and composition results in engineering of frequency dispersion, where one is able to reach different desired values of effective refractive index in the targeted range. It is also possible to localize electromagnetic field at deep subwavelength level using plasmonic nanocomposites and to ensure extreme concentrations at very small volumes [9]. A typical example of this kind of applications is nanoantennas [10-12], where one modifies geometry and composition to obtain an almost limitless range of different modes. Actually a nanoaperture can be observed as a Babinet-type complementary analogue [13] to plasmonic nanoparticle-based antenna. Strong spatial nonlocalities affect the electromagnetic behavior of the plasmonic nanocomposites. 
Since different geometrical shapes result in different mode distributions and different subwavelength field concentration levels and spatial shapes, a question is posed: under which conditions one could apply effective medium approximation (EMA) [14, 15]? In the particular case of double fishnets, the question is whether it is possible to make relatively small modifications of the aperture shape in order to obtain large changes of the effective properties. One could expect that the effects of field concentration should be largest around the sharpest corners and thus one could tune and reshape the field distribution by introducing such forms.

Changes of unit cell geometry within a fishnet structure have been reported in [16, 17]. An introduction of deeply subwavelength notches at the corners of a rectangular nanoaperture resulted in fine tuning of the effective optical properties [16].

In this paper we consider the possibility to apply rectangular patch-like extensions to the fishnet apertures, thus redistributing the electromagnetic field. In other words, we utilize compound unit cells that represent a superposition of larger and smaller rectangles. Albeit the effective geometrical and material parameters of these structures (like their aperture area and metal-to-dielectric ratio) remain very similar to those of the unperturbed initial structures, the field distribution is sharply changed. We use finite element method to investigate the amount of change thus caused to the values of the effective refractive index and permittivity and to check if this offers a possibility to tailor the effective parameters in a wider range of values.

\section{Method}

2.1. Basic Concept. The basic element of a fishnet structure, whether single-metal layer (extraordinary optical transmission array) [18], double layer (double fishnet) [19], or multiple [20], is a single subwavelength aperture. This aperture may have different shapes, including circular, elliptical, and rectangular. One can define a unit cell for a fishnet by cutting four neighboring apertures through the middle and thus defining a cross-like metal structure where a quarter of aperture is located in each corner.

In this work we modify the basic element by superposing additional apertures onto those already existing. We denote the basic aperture by the unperturbed structure, while the additional apertures are subobjects, substructures, or "patches." Both the unperturbed structure and the added substructures are simple geometrical forms like circles, rectangles, and so forth, denoted by the primitive objects or primitives. Thus one obtains a compound unit cell (a "super" unit cell) consisting of the basic object and a number of subobjects.

Figure 1 shows four situations as characteristic examples, out of a literary infinite number of possible combinations. In Figure 1(a), both the basic object and the substructures are circles; in Figure 1(b), both are hexagons. A more general case is shown in Figure 1(c), where the main object is a square, while the subobjects are octagons. The most interesting example from the practical point of view is in Figure 1(d), where the same basic shape, a square, is replicated three times to form additional deeply subwavelength corners formed by simple overlap of the main object.

One could continue to add further subobjects, making additive superstructures. Any desired form could be thus defined using simple primitive objects, making the observed situation a geometrical analog to the case of expansion of a function into series (in our case, a complex object that is expanded into primitives).

The existence of the superposed object will change the resulting geometry and thus introduce nonlocalities in its electromagnetic response. These nonlocalities will be caused by several factors, among which is the geometrical shape itself, since larger field concentrations are expected on sharper corners. Another factor is coupling between different subobjects, since narrower gaps between the subobjects result higher field localizations.

In this work we choose to analyze the case when rectangular subobjects are added to rectangular basic structures. Figure 2, left side, shows the case of an unperturbed fishnet: this is a double fishnet with rectangular nanoapertures. Figure 2, right side, shows the case when smaller rectangular subobjects are added to each corner of the basic structure.

It has been already mentioned that the effective medium theory in principle does not take into account the nonlocal nature of plasmonic resonances in complex geometries like those in the compound unit cells described here. Still, it is possible to introduce the effective parameters even for such structures as descriptive figures of merit that give insight into the spectral dispersion of their electric and magnetic response. Thus regardless of the validity of the EMA the effective parameters have a physical meaning that relates with the measured or simulated scattering parameters of the structure and helps in predicting its spectral behavior in different situations.

2.2. Extraction of Effective Electromagnetic Parameters. Here we utilize direct extraction of effective constitutive parameters (complex dielectric permittivity $\varepsilon$, permeability $\mu$, and refractive index $n$ ) from the known complex transmittance $(t)$ and reflectance $(r)$ spectra, as proposed in [21] and described in [22]. If $k_{0}$ is the free space wave number and $d$ is the total thickness of the fishnet (top and bottom layer plus dielectric), then the wave impedance that can be calculated as

$$
z= \pm \sqrt{\frac{\left(1+r+t \exp \left(j k_{0} d\right)\right)\left(1+r-t \exp \left(j k_{0} d\right)\right)}{\left(1-r-t \exp \left(j k_{0} d\right)\right)\left(1-r+t \exp \left(j k_{0} d\right)\right)}}
$$

while the effective refractive index is

$$
n=\frac{\operatorname{Im}\left\{\ln \left(e^{j n k_{0} d}\right)\right\}+2 m \pi}{k_{0} d}-j \frac{\operatorname{Re}\left\{\ln \left(e^{j n k_{0} d}\right)\right\}}{k_{0} d}
$$

where $m$ is an integer that represents the branch index of the real part of $n$ and the exponential part is

$$
\exp \left(j n k_{0} d\right)=t \exp \left(j k_{0} d\right)\left(1-r \frac{z-1}{z+1}\right)^{-1}
$$




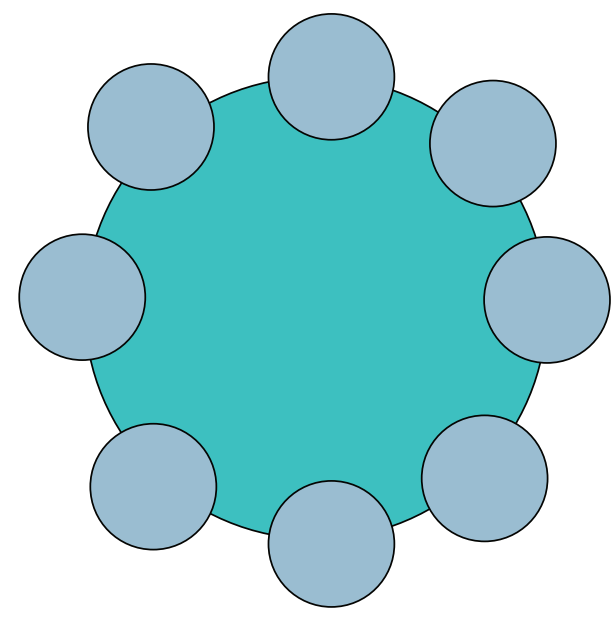

(a)

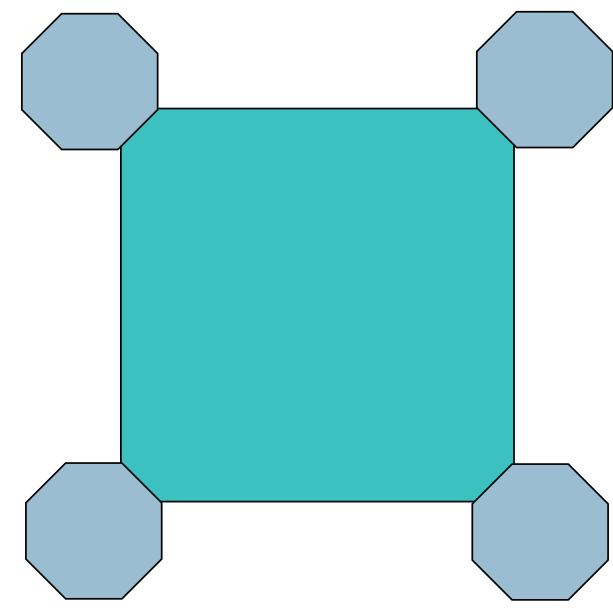

(c)

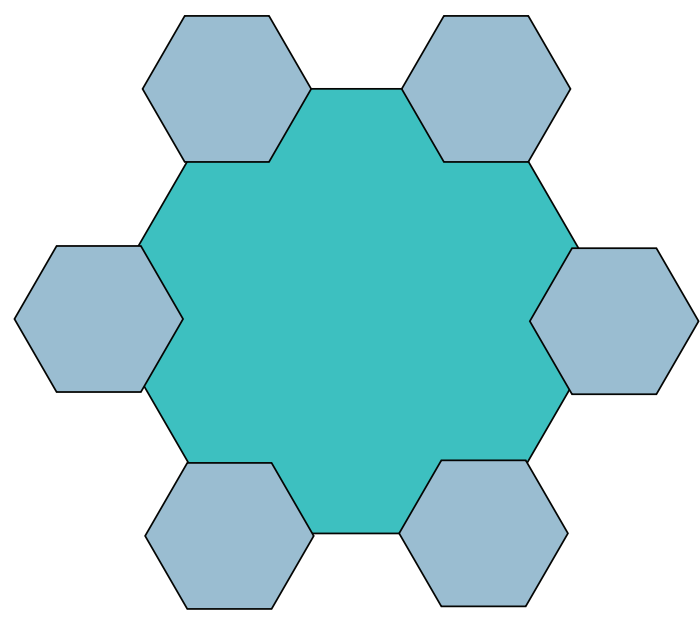

(b)

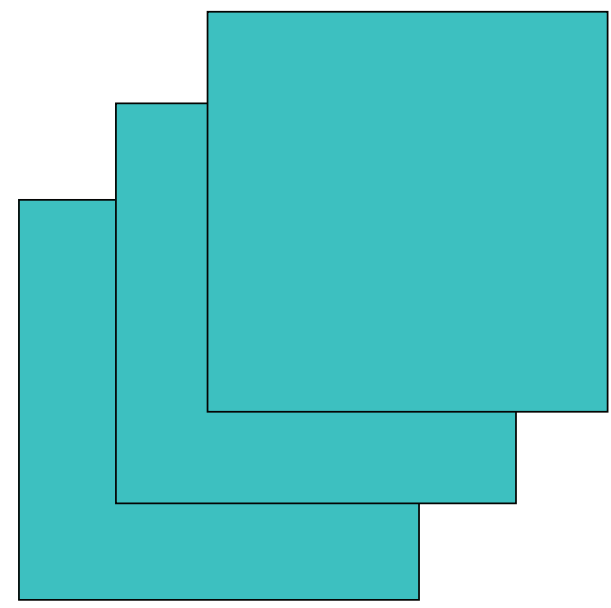

(d)

FiguRE 1: Examples of different aperture forms consisting from "primitive" subobjects. (a) Object and subobjects are circles; (b) object and subobjects are hexagons; (c) object is square, subobjects octagons; (d) only main object is replicated, subobject features are generated by intersecting these replicas.
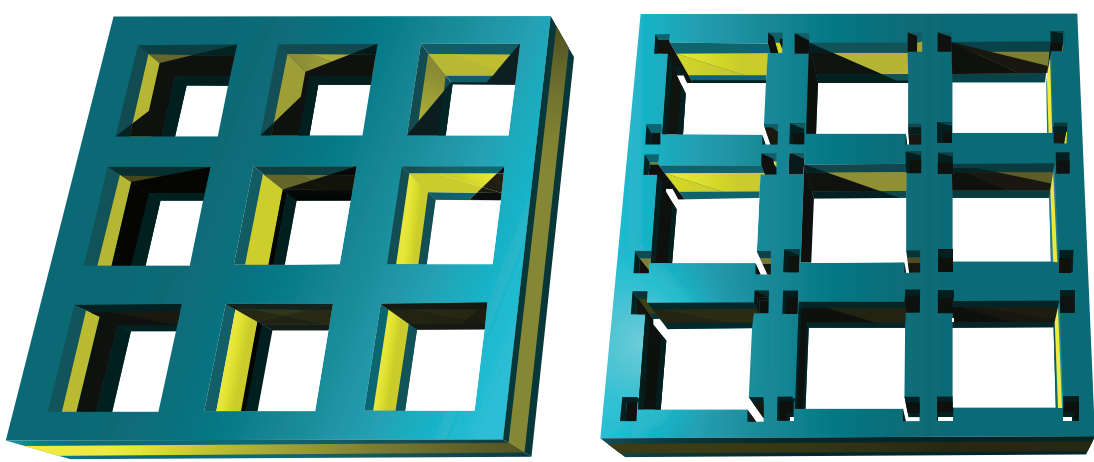

FIGURE 2: Square apertures versus compound apertures.

The values of $\varepsilon$ and $\mu$ are further calculated from the obtained wave impedance and refractive index. All expressions except the first have multiple branches and it is necessary to choose the right one with a proper sign. For lossy materials without active media the conditions are $\operatorname{Re}\{z\}>0$ and $\operatorname{Im}\{n\}>0$.
The figure of merit of a metamaterial describes its absorptive losses and is defined as the ratio between the real and imaginary part of refractive index [18]:

$$
\mathrm{FOM}=\frac{|\operatorname{Re}(n)|}{\operatorname{Im}(n)} .
$$




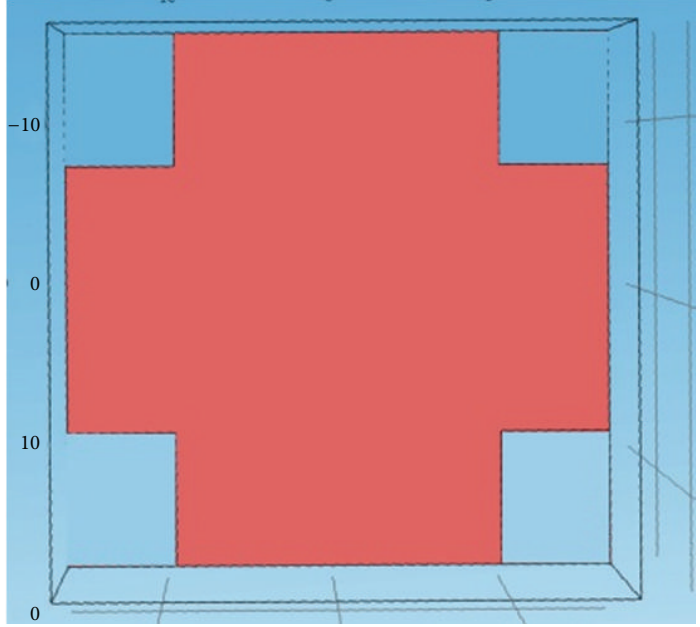

(a)

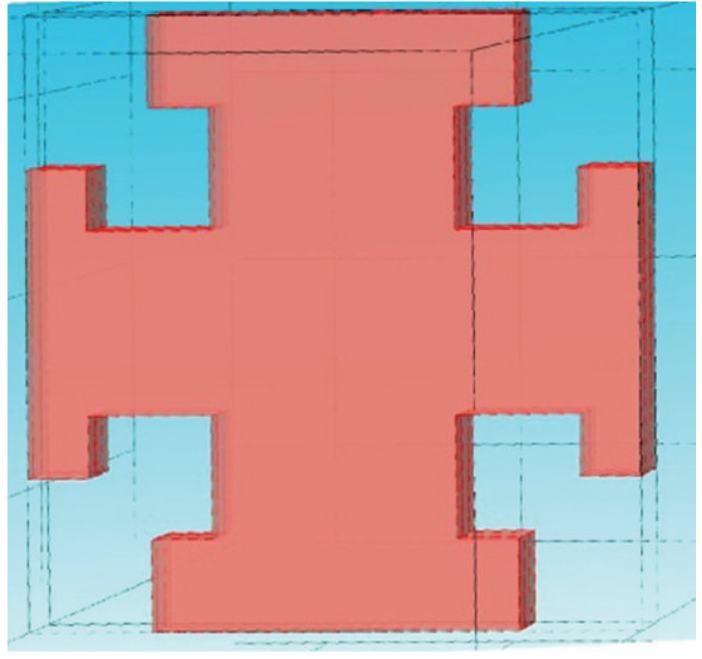

(c)

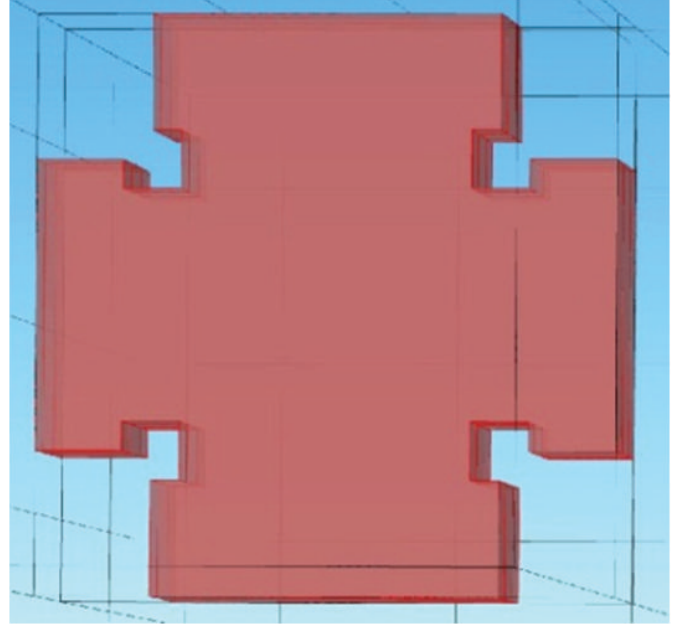

(b)

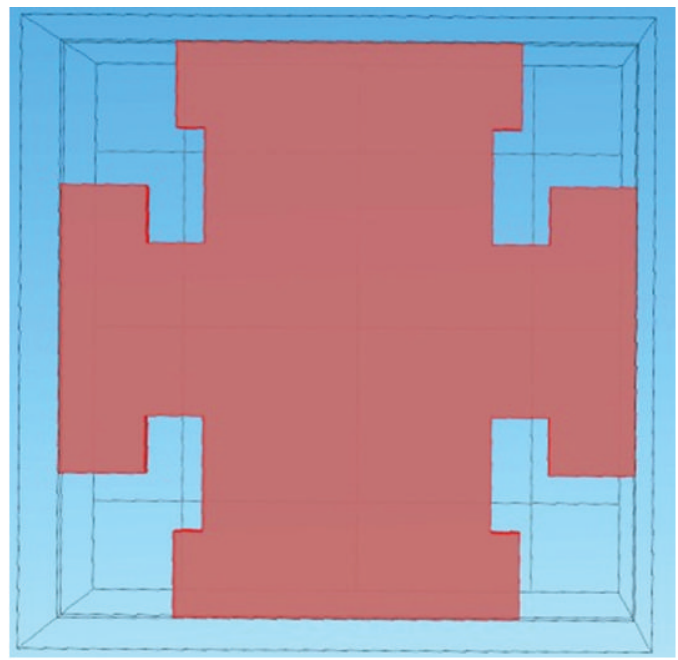

(d)

FIGURE 3: Types of unit cells considered; (a) basic (unperturbed) shape-simple rectangular apertures; (b) compound aperture (super unit cell) obtained by adding $30 \mathrm{~nm} \times 30 \mathrm{~nm}$ square patches on each corner of the basic shape; (c) super cell obtained by adding four $60 \mathrm{~nm} \times$ $60 \mathrm{~nm}$ squares; (d) supercell obtained by adding four $30 \mathrm{~nm} \times 60 \mathrm{~nm}$ rectangles.

2.3. Structures. We consider several cases. Figure 3(a) shows the basic unit cell: a cruciform structure where a quarter of an aperture is located in each corner. Figure $3(\mathrm{~b})$ shows the structure perturbed by four $30 \mathrm{~nm} \times 30 \mathrm{~nm}$ subobjects, while Figure 3(c) shows $60 \mathrm{~nm} \times 60 \mathrm{~nm}$ subobjects and Figure 3(d) shows $30 \mathrm{~nm} \times 60 \mathrm{~nm}$ subobjects. Each rectangular subobject is placed so that the intersection of its diagonals coincides with a corner of the main object, while the sides of the main object and the subobjects are parallel.

The dimensions of the basic structure were as follows: the aperture dimensions are $60 \mathrm{~nm} \times 80 \mathrm{~nm}$, square unit cell with a $300 \mathrm{~nm}$ side, the top and the bottom metal layer are $30 \mathrm{~nm}$ thick, and the thickness of the dielectric part is $40 \mathrm{~nm}$.

It has been assumed that the metal part was gold, well described by Drude model. Material parameters for gold were taken as follows: plasma frequency $\omega_{p}=7.175 \mathrm{PHz}$ and collision frequency $\gamma=6.5 \mathrm{THz}$ [23]. The dielectric part was aluminum oxide with a refractive index of 1.63 .
2.4. Numerical. We utilized Comsol Multiphysics software package ( $\mathrm{RF}$ module) to calculate the scattering parameters of our fishnets. The unit cell is treated as a waveguide surrounded by air. We applied the Perfect Electric Conductor and the Perfect Magnetic Conductor boundary conditions on the surfaces perpendicular to the respective incident fields. Thus we simulated infinite periodic structure. Two ports were added above and below the unit cell, each of them parallel to the surface. One port was active and incident light entered the waveguide through it. Parametric sweep of incident light wavelength was used to determine the spectral dependence.

\section{Results and Discussion}

Figure 4 shows the transmittance and reflectance for the unperturbed aperture shape. A sharp reflectance dip is observed between 800 and $900 \mathrm{~nm}$, as well as a high reflectance band between $600 \mathrm{~nm}$ and about $820 \mathrm{~nm}$. 


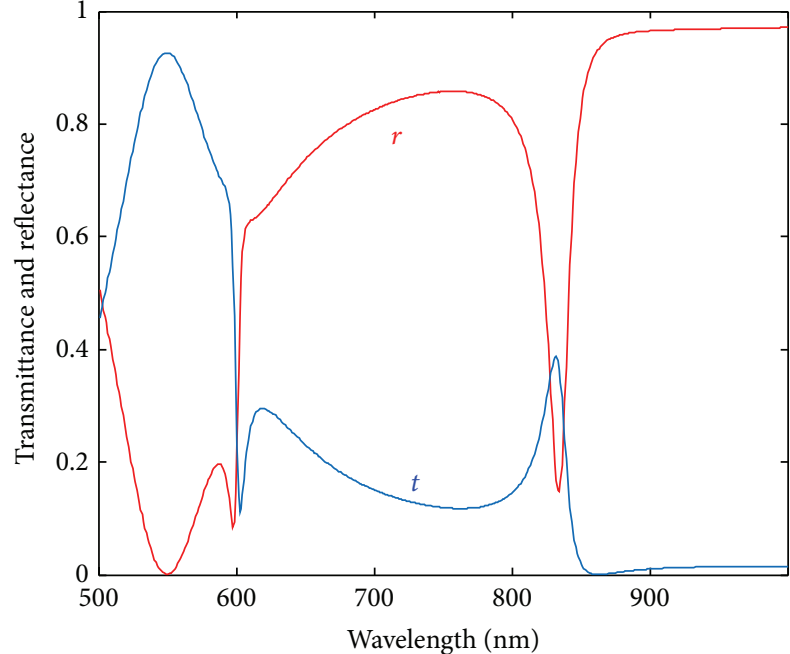

FIGURE 4: Transmittance $t$ and reflectance $r$ for the unperturbed (basic) shape.

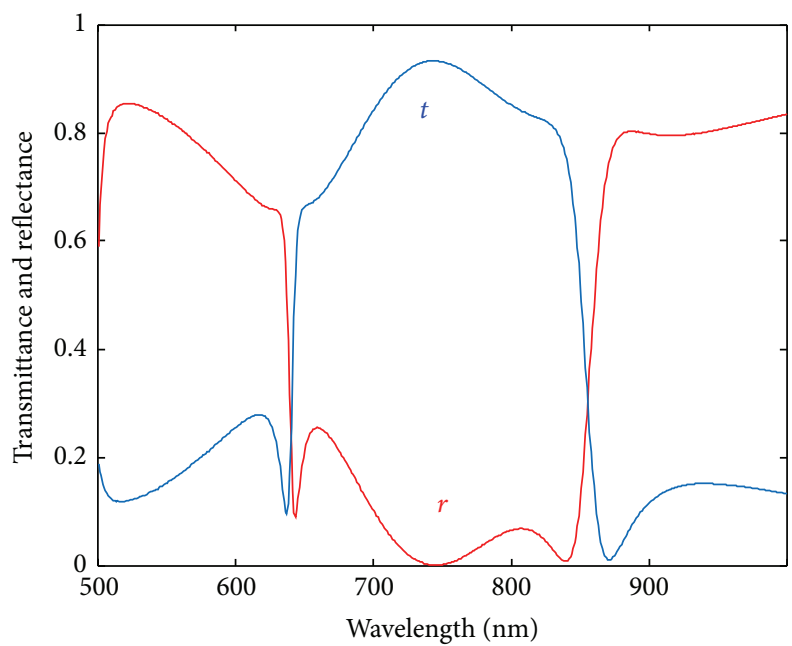

FIGURE 5: Transmittance $t$ and reflectance $r$ for the $30 \mathrm{~nm} \times 60 \mathrm{~nm}$ squares on the corners of the basic shape.

In the case when a $30 \mathrm{~nm} \times 60 \mathrm{~nm}$ subobject is added to each corner of the basic shape, a drastic change is observed in the spectra (Figure 5). A dramatic rise of transmission and drop of reflection occur where a reflection band existed. Basically a new transmission window occurs within the plasmonic bandgap.

Similar situation, but less drastic, is observed for square subobjects. For $30 \mathrm{~nm} \times 30 \mathrm{~nm}$ subobject (Figure 6) transmission is increased, but its overall level decreases with the wavelength. For $60 \mathrm{~nm} \times 60 \mathrm{~nm}$ (Figure 7) the situation is very similar, but the transmission increases with the wavelength.

Figure 8 shows the spectral absorption for the unperturbed case and the $30 \mathrm{~nm} \times 60 \mathrm{~nm}$ case. A distinct redshift is observed in the case with superposed subobjects, stronger for shorter wavelengths.

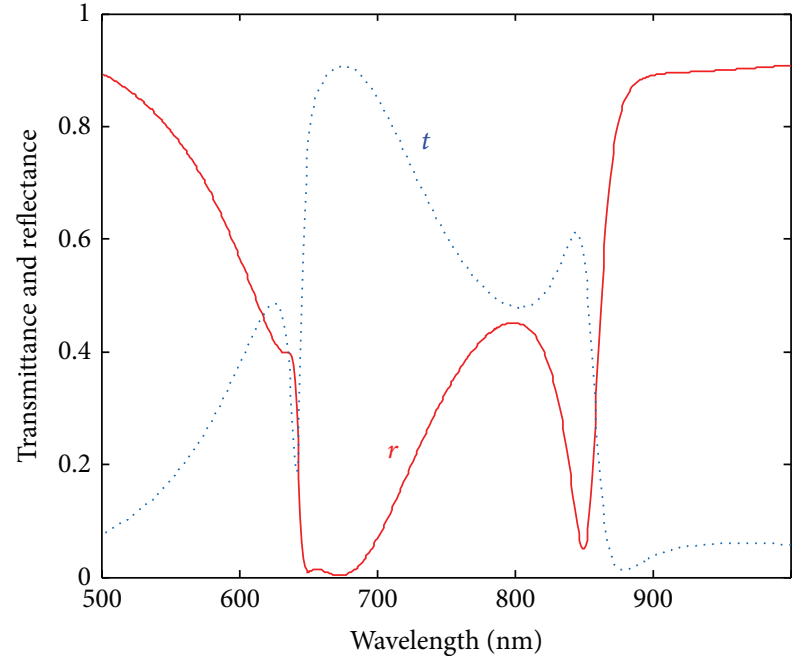

Figure 6: Transmittance $t$ and reflectance $r$ for the $30 \mathrm{~nm} \times 30 \mathrm{~nm}$ squares on the corners of the basic shape.

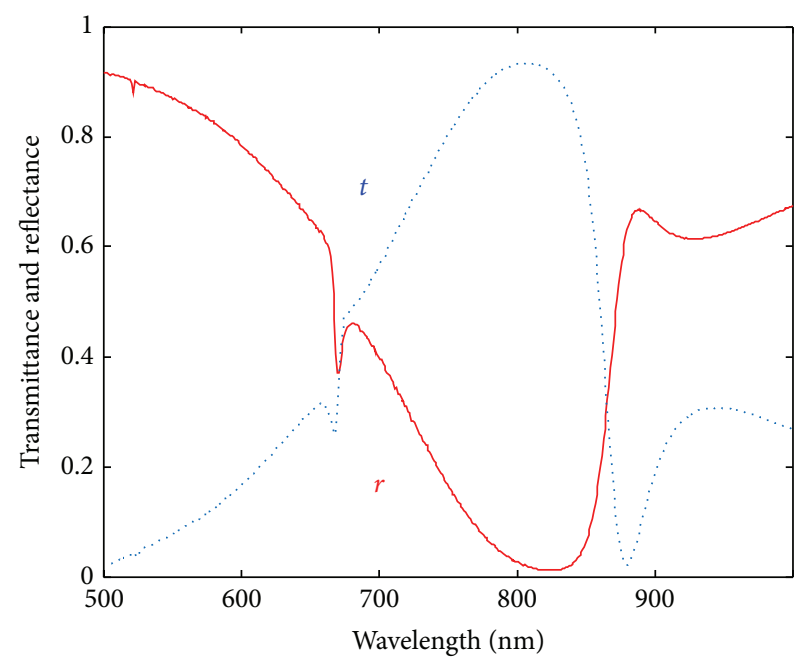

FIGURE 7: Transmittance $t$ and reflectance $r$ for the $60 \mathrm{~nm} \times 60 \mathrm{~nm}$ squares on the corners of the basic shape.

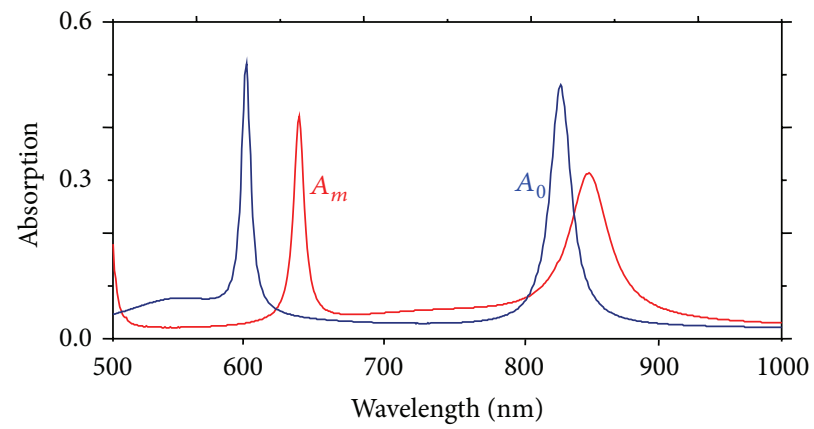

FIGURE 8: Absorption coefficients for the unperturbed aperture $\left(A_{0}\right.$, blue line) and for the one with $30 \mathrm{~nm} \times 60 \mathrm{~nm}$ subobjects $\left(A_{m}\right.$, red line).

A comparison of Figure 4 with Figures 5-7 shows that relatively small deviations from the unperturbed object cause 


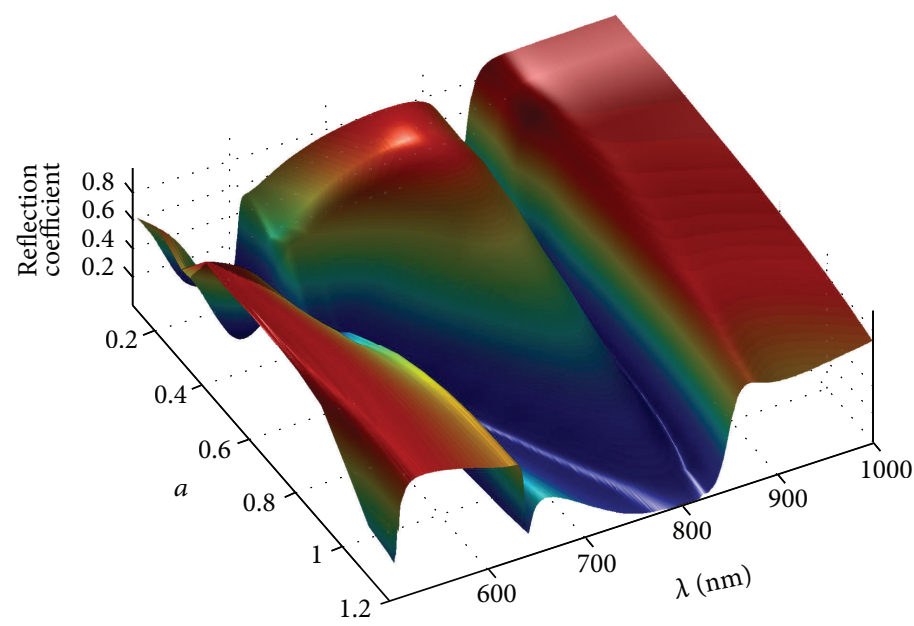

FIGURE 9: Spectral reflection coefficient of the compound unit cell-based double fishnet for rectangular corner patches. The basic patch is $30 \mathrm{~nm} \times 60 \mathrm{~nm}$, and the scaling factor for its both side lengths is $a$.

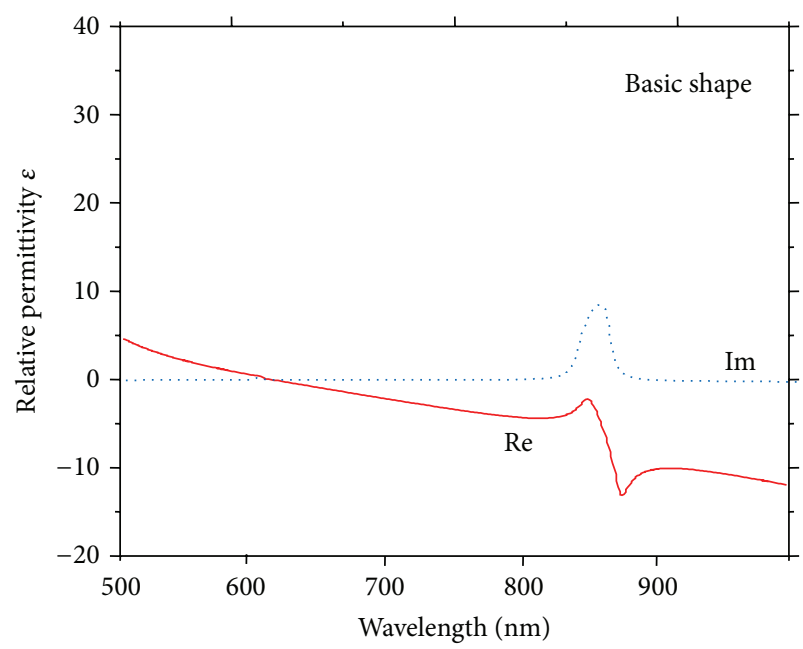

(a)

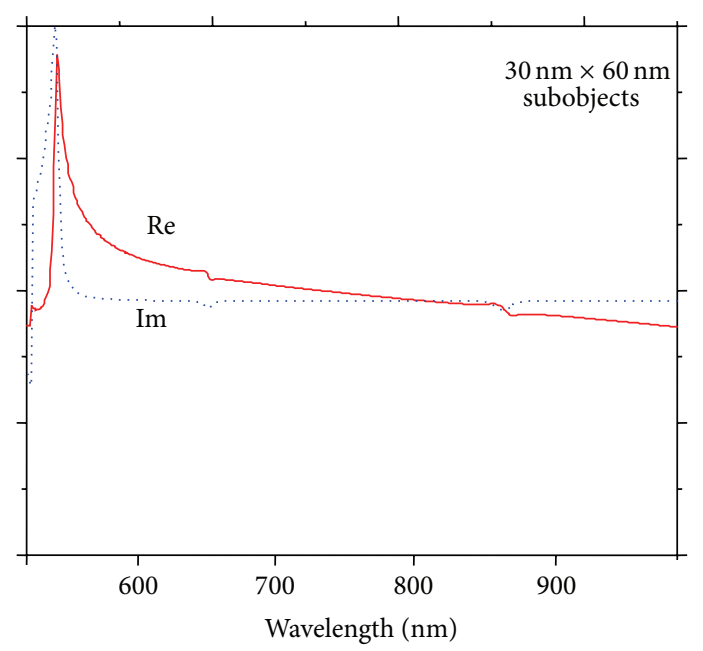

(b)

FIGURE 10: Extracted effective relative dielectric permittivity for the basic structure (a) and for the one with $30 \mathrm{~nm} \times 60 \mathrm{~nm}$ subobjects as in Figure 3(d) (b).

large changes of the resulting spectral characteristics. Such sensitivity is convenient for the use of metasurfaces and $2 \mathrm{D}$ plasmonic crystals in sensing. However, it requires a careful design of the structures and accurate fabrication methods when aiming at specific values of the resulting optical parameters. It also points out that inaccurate fabrication and the appearance of spatial noise may completely wipe out the desired optical properties, although the effective values of optical parameters will be practically unchanged. The reason for this is again the appearance of nonlocalities that may become very strong in some geometries.

We analyzed the dependence of the optical response of the structure to perturbations of the basic geometry of the unit cell and summarized our results in Figure 9. The unperturbed structure is identical to that used to obtain Figures $4-8$, but the dimensions of the corner patches are now varied. The starting corner patch used is $30 \mathrm{~nm} \times 60 \mathrm{~nm}$. We multiplied the sides of this corner patch with a scaling factor $a$ that varied from 0.1 (furnishing a corner patch with the dimensions $3 \mathrm{~nm} \times 6 \mathrm{~nm})$ to 1.2 . We can see that the highly reflective band between $600 \mathrm{~nm}$ and $800 \mathrm{~nm}$ is gradually replaced by a transmission band in the same wavelength range. At the same time, the wavelengths of its edges remain practically constant.

Figures 10-12 show the optical constants extracted by using the procedures outlined in $[21,22]$. Figure 10 (a) shows the extracted relative dielectric permittivity for the basic structure as shown in Figure 3(a), while Figure 10(b) shows the extracted permittivity for the structure with $30 \mathrm{~nm} \times$ $60 \mathrm{~nm}$ subobjects, shown in Figure 3(d). Figure 11 shows relative magnetic permeability and Figure 12 extracted effective refractive index, all for the same structures. 


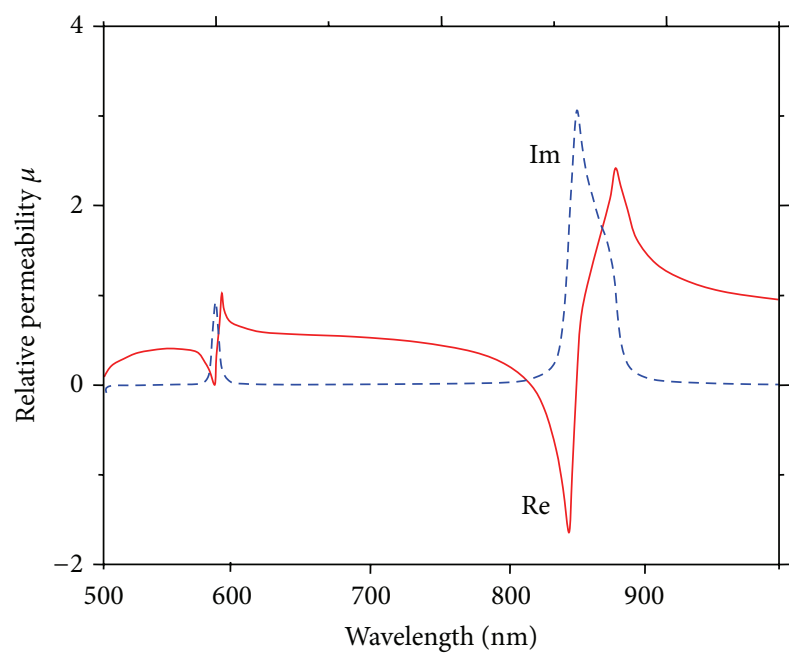

(a)

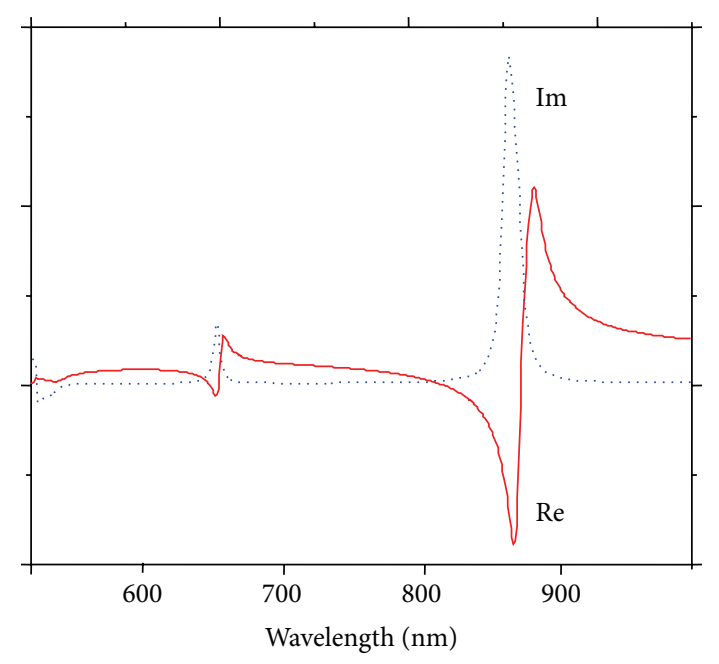

(b)

Figure 11: Extracted effective relative magnetic permeability for the basic structure (a) and for the one with $30 \mathrm{~nm} \times 60 \mathrm{~nm}$ subobjects as in Figure 3(d) (b).

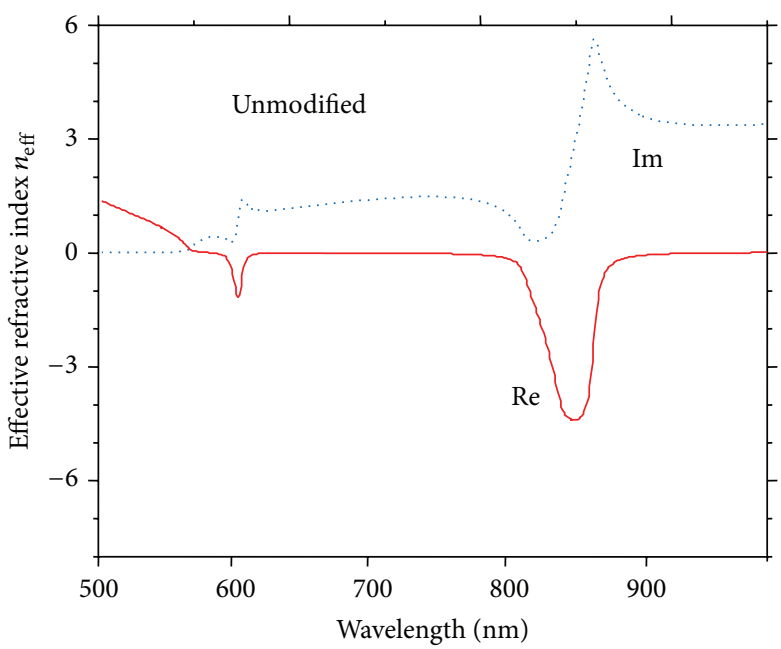

(a)

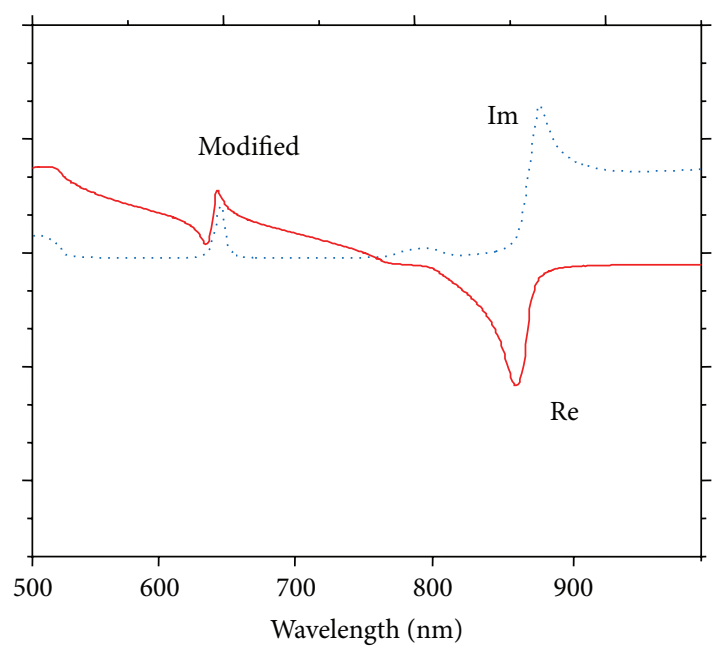

(b)

FIGURE 12: Extracted effective refractive index for the basic structure (a) and for the one with $30 \mathrm{~nm} \times 60 \mathrm{~nm}$ subobjects as in Figure 3(d) (b).

Figure 10(a) shows that in the unperturbed situation the real part of the relative dielectric permittivity (notation: Re) becomes negative for wavelengths above $600 \mathrm{~nm}$. At the resonant frequencies between $800 \mathrm{~nm}$ and $900 \mathrm{~nm}$ there is the characteristic increase of the absorption losses, as seen from the peak in the imaginary component of the permittivity (notation Im). This behavior is actually similar to that of metallic structures, however contrary to metals; it is possible here to tune the spectral dispersion through choosing the aperture geometry. This is seen in Figure 10(b), where the peaks of both the real and imaginary component are shifted to lower wavelengths.

Figure 11 shows the real and imaginary component of effective permeability. This value should be understood as a parameter describing high-frequency magnetic response of the metal-dielectric nanocomposite under consideration. Here too one can observe negative values of the real part and an increase of the imaginary component connected with higher losses.

Figure 12 shows the effective refractive index as the synthetic parameter obtained from the effective permittivity and permeability. Since there is a range of overlapping negative values of the real parts of $\varepsilon$ and $\mu$, this means that there will be a range of negative effective refractive index values; that is, the structure will behave as negative index metamaterial. This behavior is qualitatively preserved in the modified structure as well. However, one should note a beneficial property of the modified structure: it has lower losses (i.e., smaller imaginary part of effective refractive index) compared to the unmodified one. This can be also seen in Figure 13 that shows 


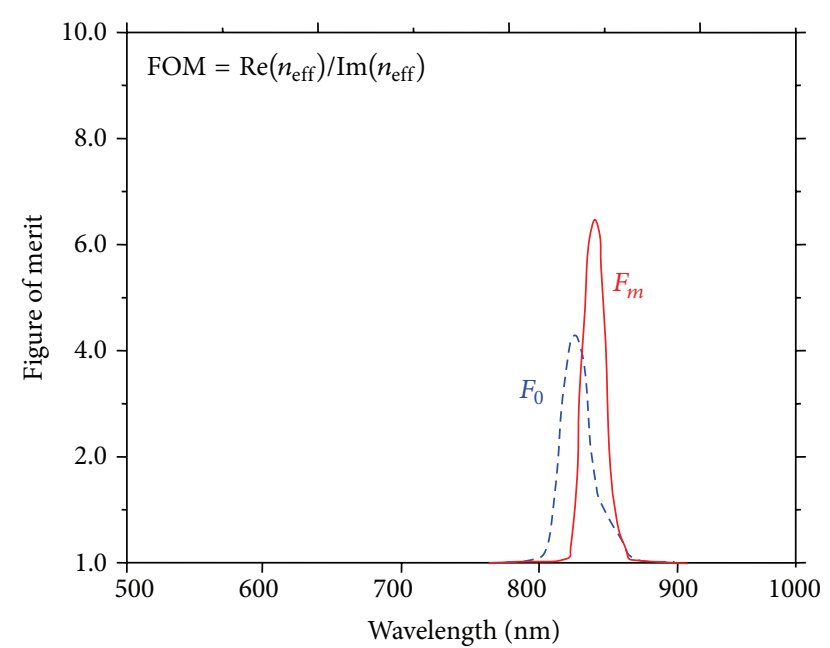

FIGURE 13: Comparison of figures of merit for the basic structure (dashed blue lines) and for the one with $30 \mathrm{~nm} \times 60 \mathrm{~nm}$ subobjects (solid red line).

the comparison of figures of merit between the unperturbed case and the case perturbed with $30 \mathrm{~nm} \times 60 \mathrm{~nm}$ rectangles. The value of the obtained figure of merit is about $50 \%$ larger than in the unperturbed case.

It is important to mention here some technological aspects of the described approach. Since the main objects have subwavelength dimensions, the subobjects must be deeply subwavelength, and both are intended for fabrication by the top-down approach. With the main objects in the dimension range of several hundreds of nanometers, the subobjects have dimensions of tens of nanometers. Having in mind the limitations of the state-of-the-art lithographic equipment, it is obviously rather difficult to reproduce such shapes.

We argue that one possible solution is to generate supercells using identical overlapping objects, an example being the one shown in Figure 1(d). A square with the dimensions comparable to the wavelength and thus more easily delineated is replicated there three times, each time with a slight misplacement. The newly created compound structure contains new deeply subwavelength features created at the intersections of the overlapping "big" objects. These new features ensure new hotspots of electromagnetic field and thus cause reshaping of the optical spectra.

The procedure obviously cannot create objects like those in Figures 1(a) to 1(c), which contain smaller subobjects. However, it does create sharp corners as a result of addition of identical objects. Alternatively, it can replicate the main object twice at a subwavelength distance, thus ensuring the appearance of a field hotspot within the gap.

The main object (which can also be circular, etc.) could be thus used to pattern a photolithographic mask at the maximum available resolution of the existing equipment. This resolution is obviously insufficient to get any deeply subwavelength features. As the next step, however, we use alignment equipment to simply shift the mask and repeat pattern definition on the substrate. In this way the shapes like those in Figure 1(d) can be generated, ensuring the appearance of sharp corners. Thus we reduce the problem of resolution of lithographic masks to the problem of alignment of the existing lower resolution masks.

\section{Conclusion}

We constructed 2D arrays of nanoapertures in double fishnet plasmonic configuration with complex $2 \mathrm{D}$ shapes on deep subwavelength level. To define the aperture geometry we applied a simple additive approach, where an initial form is modified by overlapping it with additional objects/subobjects. In this manner one can use a set of primitives to generate additional nonlocalities without increasing the complexity of the system or requiring finer resolutions, which contributes to a lower fabrication cost. We described a possible approach to the fabrication of some of such objects, which is to simply shift the same photolithographic mask and repeat its pattern, where field localizations could be expected near the deeply subwavelength corners at the intersection points of the "large" objects.

We show that the nonlocalities introduced by overlapping simple objects can be used to produce large deviations of spectral dispersion from the unperturbed case even in the case of relatively minor additive modifications of the basic geometry.

Our approach points out to the possibility to tailor the dispersion and the resonance spectra for plasmonic nanocomposites by adjusting the local geometry and exploiting localized interactions at a subwavelength level. Additional resonances can be introduced and spectral dispersion can be shifted and tuned up to a desired level. The obtained freedom in tailorability could be used to fabricate structures for plasmonic sensing of chemical or biological analytes and generally for the fabrication of planar waveguides utilizing two-dimensional plasmonic crystals.

\section{Conflict of Interests}

The authors declare that there is no conflict of interests regarding the publication of this paper.

\section{Acknowledgment}

The paper is a part of the research funded by the Serbian Ministry of Education, Science and Technological Development within the Project TR32008.

\section{References}

[1] M. Kafesaki, I. Tsiapa, N. Katsarakis, T. Koschny, C. M. Soukoulis, and E. N. Economou, "Left-handed metamaterials: the fishnet structure and its variations," Physical Review BCondensed Matter and Materials Physics, vol. 75, no. 23, Article ID 235114, 2007.

[2] S. Zhang, W. Fan, N. C. Panoiu, K. J. Malloy, R. M. Osgood, and S. R. J. Brueck, "Experimental demonstration of near-infrared negative-index metamaterials," Physical Review Letters, vol. 95, no. 13, Article ID 137404, 2005. 
[3] Z. Jakšić, D. Tanasković, and J. Matović, "Fishnet-based metamaterials: spectral tuning through adsorption mechanism," Acta Physica Polonica A, vol. 116, no. 4, pp. 333-335, 2009.

[4] T. W. Ebbesen, H. J. Lezec, H. F. Ghaemi, T. Thio, and P. A. Wolff, "Extraordinary optical transmission through sub-wavelenght hole arrays," Nature, vol. 391, no. 6668, pp. 667-669, 1998.

[5] J. B. Pendry, L. Martín-Moreno, and F. J. Garcia-Vidal, "Mimicking surface plasmons with structured surfaces," Science, vol. 305, no. 5685, pp. 847-848, 2004.

[6] A. G. Brolo, R. Gordon, B. Leathem, and K. L. Kavanagh, "Surface plasmon sensor based on the enhanced light transmission through arrays of nanoholes in gold films," Langmuir, vol. 20, no. 12, pp. 4813-4815, 2004.

[7] W. Cai and V. Shalaev, Optical Metamaterials: Fundamentals and Applications, Springer, Dordrecht, The Netherlands, 2009.

[8] S. A. Ramakrishna and T. M. Grzegorczyk, Physics and Applications of Negative Refractive Index Materials, SPIE Press, Bellingham, Wash, USA; CRC Press; Taylor \& Francis, Boca Raton, Fla, USA, 2009.

[9] A. Polman and H. A. Atwater, "Photonic design principles for ultrahigh-efficiency photovoltaics," Nature Materials, vol. 11, no. 3, pp. 174-177, 2012.

[10] P. Biagioni, J.-S. Huang, and B. Hecht, "Nanoantennas for visible and infrared radiation," Reports on Progress in Physics, vol. 75, no. 2, Article ID 024402, 2012.

[11] A. Alu and N. Engheta, "Theory, modeling and features of optical nanoantennas," IEEE Transactions on Antennas and Propagation, vol. 61, no. 4, pp. 1508-1517, 2013.

[12] S. V. Boriskina, H. Ghasemi, and G. Chen, "Plasmonic materials for energy: from physics to applications," Materials Today, vol. 16, no. 10, pp. 375-386, 2013.

[13] F. Falcone, T. Lopetegi, M. A. G. Laso et al., "Babinet principle applied to the design of metasurfaces and metamaterials," Physical Review Letters, vol. 93, no. 19, 2004.

[14] T. Koschny, M. Kafesaki, E. N. Economou, and C. M. Soukoulis, "Effective medium theory of left-handed materials," Physical Review Letters, vol. 93, no. 10, Article ID 107402, 2004.

[15] P. Lalanne and D. Lemercier-Lalanne, "On the effective medium theory of subwavelength periodic structures," Journal of Modern Optics, vol. 43, no. 10, pp. 2063-2085, 1996.

[16] Z. H. Jiang, S. Yun, L. Lin, J. A. Bossard, D. H. Werner, and T. S. Mayer, "Tailoring dispersion for broadband low-loss optical metamaterials using deep-subwavelength inclusions," Scientific Reports, vol. 3, article 1571, 2013.

[17] Z. H. Jiang, L. Lin, J. A. Bossard, and D. H. Werner, "Bifunctional plasmonic metamaterials enabled by subwavelength nano-notches for broadband, polarization-independent enhanced optical transmission and passive beam-steering," Optics Express, vol. 21, no. 25, pp. 31492-31505, 2013.

[18] T. W. Ebbesen, H. J. Lezec, H. F. Ghaemi, T. Thio, and P. A. Wolff, "Extraordinary optical transmission through sub-wavelenght hole arrays," Nature, vol. 391, no. 6668, pp. 667-669, 1998.

[19] M. Kafesaki, I. Tsiapa, N. Katsarakis, T. Koschny, C. M. Soukoulis, and E. N. Economou, "Left-handed metamaterials: the fishnet structure and its variations," Physical Review B: Condensed Matter and Materials Physics, vol. 75, no. 23, Article ID 235114, 2007.

[20] J. Valentine, S. Zhang, T. Zentgraf et al., “Three-dimensional optical metamaterial with a negative refractive index," Nature, vol. 455, no. 7211, pp. 376-379, 2008.
[21] D. R. Smith, S. Schultz, P. Markoš, and C. M. Soukoulis, "Determination of effective permittivity and permeability of metamaterials from reflection and transmission coefficients," Physical Review B-Condensed Matter and Materials Physics, vol. 65, no. 19, Article ID 195104, 2002.

[22] G. Parisi, Propagation of electromagnetic waves in "Fishnet" metamaterials [Doctoral thesis], Università Degli Studi di Padova, Padua, Italy, 2012.

[23] E. D. Palik, Ed., Handbook of, Optical Constants of Solids, Academic Press, London, UK, 1998. 

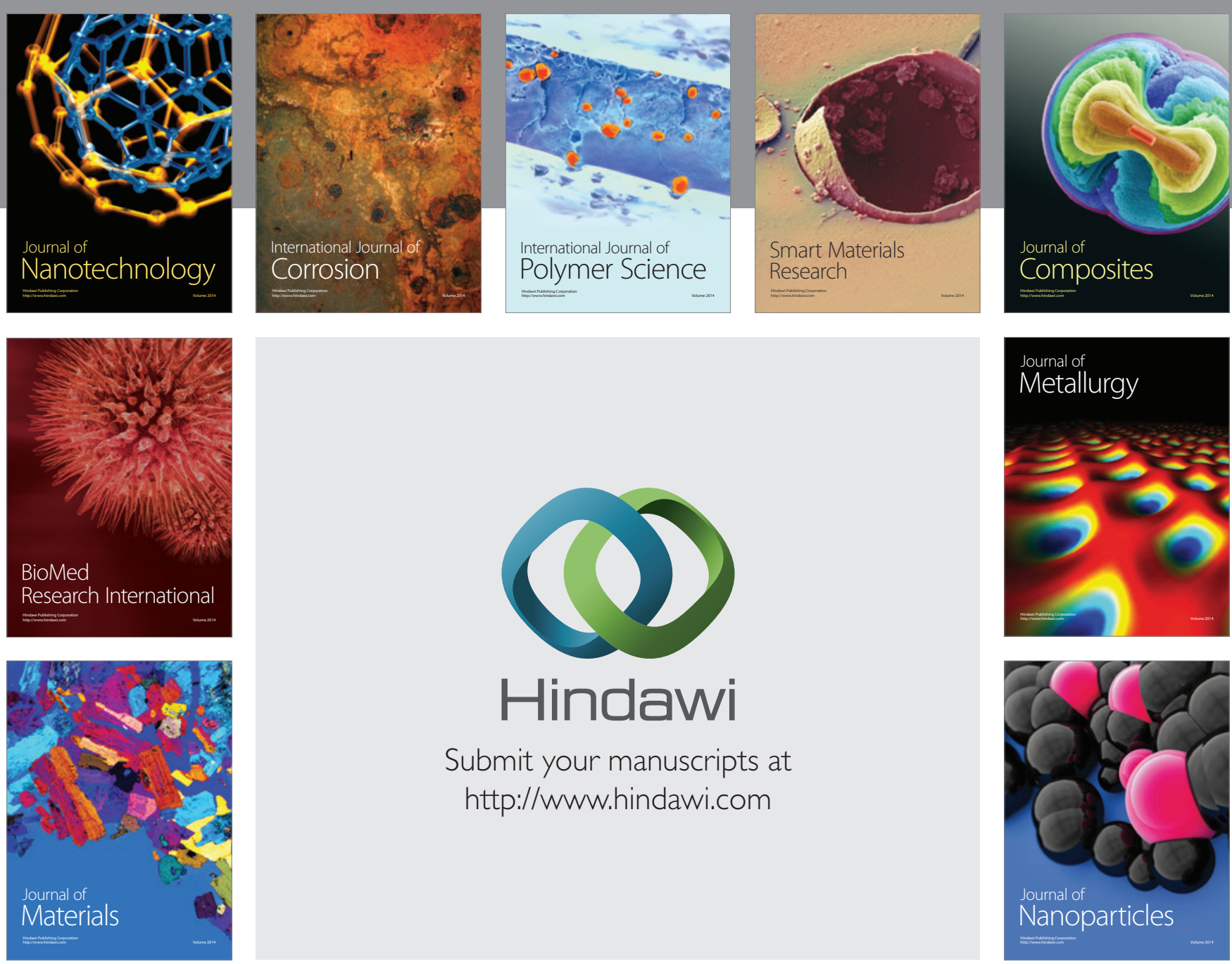

Submit your manuscripts at http://www.hindawi.com
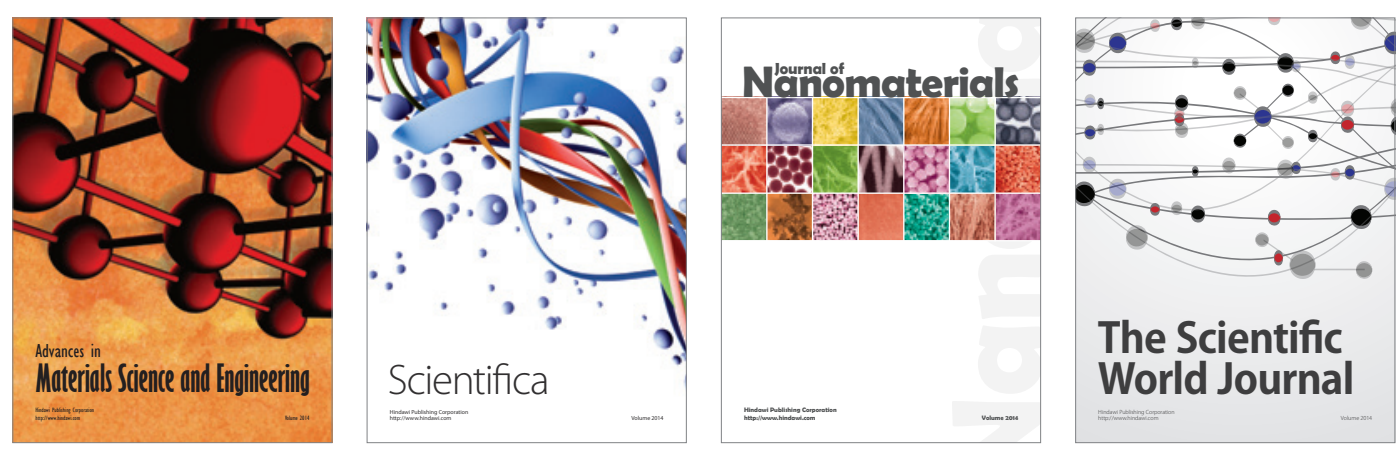

\section{The Scientific World Journal}
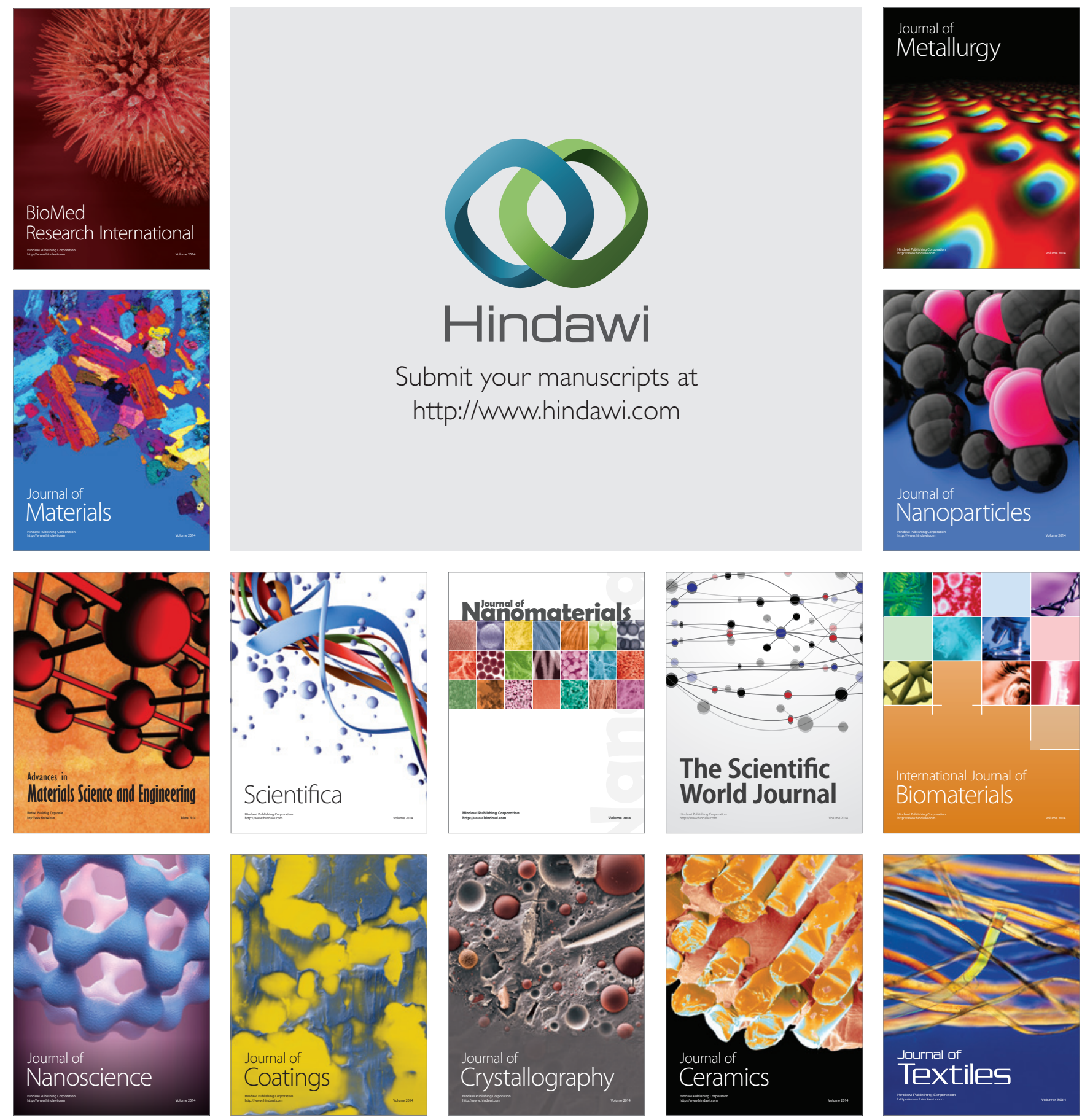